\title{
Is a $1 \%$ isolated coronary artery bypass grafting mortality really a valid goal?
}

Richard Lee, MD, MBA

See related article on pages 2686-96.

"The Challenge of Achieving 1\% Operative Mortality for Coronary Artery Bypass Grafting: A Multi-institution Society of Thoracic Surgeons Database Analysis," by LaPar and colleagues in this issue of the Journal, is a sophisticated analysis that uses hierarchic multiple regression models in a population of 34,416 patients undergoing isolated primary coronary artery bypass grafting (CABG) at the 17 Virginia Cardiac Surgery Quality Initiative institutions during an 11-year period. In the article, LaPar and colleagues explore the feasibility of obtaining $1 \%$ mortality for patients in this population.

In short, they conclude that achieving $1 \%$ mortality is not likely in the entire population. By means of the Society of Thoracic Surgeons Predicted Risk of Mortality (PROM), they have determined that a mortality of less than $1 \%$ can be achieved when the PROM is at or below $1.27 \%$. This includes $57 \%$ of the population.

This article is an important start to inevitable discussions in our challenging and evolving health care environment. As a cardiothoracic community, we have led the field of medicine in measures of outcome and used them for quality improvement. As such, we are, at times, victims of our own success. The use of our data for publically available reporting allows patients to choose a surgeon and institution that offers the best outcome. I hope that we all believe that this is a public service. As this article suggests, however, even with adjusted risk, at times the data are unreliable. In particular, the PROM scores were inaccurate above $25 \%$. Moreover, in the high-risk patients, when PROM was $4.6 \%$ or above, the decedents had striking differences in risk profiles that included preoperative dialysis, heart failure, and emergency surgery. LaPar and colleagues go so far as to suggest that surgeons performing $\mathrm{CABG}$

\footnotetext{
From the Department of Cardiovascular Medicine and Surgery, St Louis University, St Louis, Mo.

Disclosures: Author has nothing to disclose with regard to commercial support.

Received for publication Sept 24, 2014; accepted for publication Sept 25, 2014; available ahead of print Oct 18, 2014.

Address for reprints: Richard Lee, MD, MBA, Cardiovascular Medicine and Surgery, St Louis University, 3635 Vista Ave, 13th Floor DT, St Louis, MO 63110 (E-mail: rlee@slu.edu).

J Thorac Cardiovasc Surg 2014;148:2697-8

$0022-5223 / \$ 36.00$

Copyright (c) 2014 by The American Association for Thoracic Surgery

http://dx.doi.org/10.1016/j.jtcvs.2014.09.092
}

operations in these select populations should not be penalized for disproportionally high mortalities.

With indiscriminate public emphasis on mortality, even risk adjusted, the inevitable surgical response will be to move toward zero mortality. If the target arbitrarily is placed at $1 \%$ mortality, this may lead to rationing of care in the high-risk patient population. LaPar and colleagues suggest that a $1 \%$ mortality goal in only reasonable for approximately $60 \%$ of patients, leaving potentially $40 \%$ of patients at risk for restriction of care. Clearly, this bar is too high.

There are several limitations in the article that highlight challenges with quality reporting. This study does not address morbidity. Although there may be a correlation, at times there may be a trade-off. There are some devastating complications of cardiac surgery that some patients would find equivalent to mortality. In addition, surgical volume was initially only measured by isolated CABG procedures. Subsequent analysis confirmed that it was correlated with total cardiac volume. This is, however, a potential pitfall in public reporting. For example, a surgeon who performs only 1 isolated CABG procedure per week but 4 combined $\mathrm{CABG}$ and valve procedures should likely be comparable to a surgeon who performs 5 CABG procedures per week. In that vein, total surgeon experience was not available. The number of lifetime operations is also an important consideration. Lastly, fewer deceased patients received discharge medicines, and this was discussed as a process improvement opportunity. This again highlights a pitfall of traditional reporting and indiscriminate emphasis on secondary end points, such as administration of specific medicines before discharge. Likely, the medicines were inappropriate in many of these cases. For example, it makes no sense to start a $\beta$-blocker for someone already receiving a $\beta$-agonist. A patient receiving inotropes after surgery is less likely to be a survivor. The same is true for an antihypertensive agent in a patient with low blood pressure. A completely low-risk, healthy patient with a good operation is likely to receive all of his or her medication. A patient who is bleeding or one with low platelets from heparin-induced thrombocytopenia is unlikely to receive aspirin. Even our sophisticated database is unlikely to be able to determine whether the medication is the cause or the effect of an untoward event in some patients. Overemphasis on specific medications inappropriately misplaces our focus off target.

Accurate measures of quality are extremely complex. Public reporting systems such as LeapFrog and HealthGrades 
tend to attempt to distill the metric to something that a layperson can understand, such as a "grade" or a "mortality number." We know that this type of metric can be deceptive and inaccurate. As thoracic surgeons, we have developed a culture of innovation, not only in the treatment of patients but also in the areas of measuring and reporting outcomes. Our next frontier should focus on developing a reporting system that is more accurate and encompassing. We should work with the existing popular reports and help with modifications. In addition, we should help educate the public as to the strengths and weaknesses of the reporting system. As a community, our goal has always been to improve quality. This article highlights the fact that we need to be more active participants in describing quality in a manner that does not lead to restriction of reasonable care. Moreover, we should be thoughtful and use the data to set new goals, rather than arbitrarily setting goals only to find out that they cannot be achieved. Reports like these demonstrate that we are in the "pole position" in data-driven quality reforms. We have yet another opportunity to stay in the lead. 